\title{
Mixing-length parameter from binaries and clusters
}

\author{
Mutlu Yıldız \\ Ege University, Dept. of Astronomy and Space Sciences, Bornova, 35100 Izmir, Turkey \\ email: mutlu.yildiz@ege.edu.tr
}

\begin{abstract}
Mixing length parameter of the mixing length theory is a free parameter and used to calibrate radii of late-type stars. From the Hyades cluster and the $\alpha$ Centauri system, we find that the parameter depends on the stellar mass and some other properties of stars. Considering some other systems, we describe how the parameter depends on stellar parameters.
\end{abstract}

Keywords. convection, stars: interiors, stars: evolution, stars: late-type, binaries: eclipsing, stars: individual ( $\alpha$ Cen, FL Lyr, V442 Cyg)

\section{Introduction}

Brightness and size of a star essentially depend on physical conditions in its nuclear core. For radius, another important issue is energy transport mechanism in outer regions. For the early-type stars, whose envelope is in radiative equilibrium, at least near the zero-age main-sequence (ZAMS), radius is determined by the heavy element abundance. For the late type stars, however, the energy transport mechanism is convection (BohmVitense, 1958) and value of the mixing-length parameter plays an essential role for the size of their models. The value of mixing-length parameter is customarily taken as the value derived from the solar calibration, despite no strict clues for the constancy.

The disagreement between observed and model radii of late-type stars in eclipsing binaries is a long-standing problem. Popper (1997), for example, discusses many of possible reasons. In many studies on stellar interior variable mixing-length parameter is also considered as a solution for such a disagreement (see for exapmle, Lebreton et al. 2001).

In this talk, we discuss how the mixing-length parameter should depend on the stellar variables in order to fit the model values of physical properties to the observed values.

\section{The mixing-length parameter from the Hyades open cluster}

Recently, stellar mass dependence of the mixing-length parameter is derived for the stars in the Hyades open cluster by Ylldız et al. (20006). In order to fit the radii (or colors) of models to the observed values, the mixing-length parameter should be an increasing function of stellar mass:

$$
\alpha=9.19\left(M / M_{\odot}-0.74\right)^{0.053}-6.65 .
$$

The age of Hyades is about $700 \mathrm{Myr}$ and it is very short time in comparison with the MS life time. Therefore this relation can be considered as the stellar mass dependence of the mixing length parameter near the ZAMS.

In deriving the expression given in Eq. 2.1, together with fundamental properties of binary stars (V818 Tau, 70 Tau, HD 27149 and $\theta^{2}$ Tau) in Hyades, slopes of lower and upper parts of MS are also used. The slope of the lower part, which contains the 
components of V818 Tau, is obtained as

$$
S_{M S 1}=\frac{\Delta M_{V}}{\Delta(B-V)}=4.6,
$$

from application of a least-square method to the data given by de Bruijne et al. (2001). For the upper part, which contains the components of $70 \mathrm{Tau}$,

$$
S_{M S 2}=\frac{\Delta M_{V}}{\Delta(B-V)}=6.6 .
$$

Very similar slopes are found also from the MS of Praesepe, which is very similar to Hyades in many respects. This means that similar difference between models and the stars also exist for this cluster.

\section{The mixing-length parameter from $\alpha$ Centauri}

We have relatively very accurate both seismic and non-seismic data for $\alpha$ Cen $\mathrm{A}$ and B. The compatibility of these two data sets is discussed in detail by Miglio and Moltaban (2005) and Ylldı (2007) While the seismic constraints imply that age of the system is about 5.6-5.9 Gy, the non-seismic constraints give 8.9 Gy. The values of the mixing-length parameters for both components are rather different. While $\alpha_{\mathrm{A}}$ is larger than $\alpha_{\mathrm{B}}$ for the non-seismic constraints, for the seismic constraints, $\alpha_{\mathrm{B}}$ is larger than $\alpha_{\mathrm{A}}$. The former result is compatible with Eq. 2.1, the latter result, however, is possible if mixing-length parameter changes with evolution (time). The mixing-length parameter can be written as functions of stellar parameters, which changes with time. Yıldız et al. (2006) give two different expressions for $\alpha$ :

$$
\begin{gathered}
f_{1}=2.5-\rho_{\mathrm{bcz}}\left(\frac{2.7}{T_{6, \mathrm{bcz}}}\right)^{4}-\rho_{\mathrm{ph}}\left(\frac{1.4}{T_{5, \mathrm{ph}}}\right)^{4}, \\
f_{2}=\frac{3.25}{\rho_{\mathrm{ph}}^{0.8}}\left(\frac{m_{\mathrm{bcz}}}{r_{\mathrm{bcz}}^{2}}\right)^{0.5}\left(\frac{T_{6, \mathrm{bcz}}}{3.8}\right)^{4} .
\end{gathered}
$$

The subscripts bcz and ph represent the base of convective zone and the photosphere, respectively. The radius of the base of the convective zone $\left(r_{\mathrm{bcz}}\right)$ and the mass inside the sphere with this radius $\left(M_{\mathrm{bcz}}\right)$ are in solar units. Both of these expressions are in good agreement with Eq. 2.1 near the ZAMS. In the later phase of MS evolution of $\alpha$ Cen A, however, $f_{1}$ is an increasing function of time but $f_{1}$ is an decreasing function.

\section{The mixing-length parameter from the eclipsing binary FL Lyr}

In order to test variability of the mixing-length parameter in some other systems, double lined eclipsing binaries with late type components are among the most suitable systems. One of these sytems is FL Lyr. Masses and radii of its components are accurately determined (Andersen 1991) from light curve and radial velocity. The Fundamental properties of the component stars are listed in Table 1. Age of the system is from Pols et al. (1997) and $Z$ is computed from calibration of photometric properties by using bolometric correction tables of Lejeune et al. (1998).

We construct models for these stars with variable mixing-length parameter and try to fit the models to the fundamental properties derived from light curve and radial velocity curve. In the calibration process, for a given hydrogen abundance $X$, we obtain 
Table 1. The properties of FL Lyr A and B (Andersen 1991). The age is from Pols et al. (1997). The metallicity is computed from the calibration of photometric properties by using bolometric correction tables of Lejeune et al. (1998).

\begin{tabular}{|c|c|c|c|c|c|c|c|c|c|}
\hline Star & $B-V$ & $\log \left(M / M_{\odot}\right)$ & $\log \left(R / R_{\odot}\right)$ & $\log (g)$ & $\log \left(T_{\text {eff }}\right)$ & $\log (L / L \odot)$ & $M_{V}$ & $\operatorname{age}(y)$ & $Z$ \\
\hline $\mathrm{A}$ & 0.52 & $1.218 \pm 0.016$ & $1.282 \pm 0.028$ & $4.308 \pm 0.020$ & $3.789 \pm 0.007$ & $0.32 \pm 0.03$ & $3.98 \pm 0.09$ & $2.37 \mathrm{e} 9$ & 0.0177 \\
\hline B & 0.78 & $0.958 \pm 0.012$ & $0.962 \pm 0.028$ & $4.453 \pm 0.026$ & $3.724 \pm 0.008$ & $-0.18 \pm 0.04$ & $5.40 \pm 0.10$ & $2.37 \mathrm{e} 9$ & 0.0187 \\
\hline
\end{tabular}

Table 2. For given initial hydrogen abundance $(\mathrm{X}=0.70)$ and $\alpha$ values $\left(\alpha_{\mathrm{A}}=\alpha_{\mathrm{B}}=1.82\right)$, time $t$ is found for FL Lyr A and $\mathrm{B}$, at which luminosity of model is equal to the observed value. The logarithmic time difference $\left(t_{\mathrm{A}}-t_{\mathrm{B}}\right) / t_{\mathrm{A}}$ for different values of $\mathrm{Z}$ is given in the fourth column.

\begin{tabular}{rrrr}
\hline $\mathrm{Z}$ & $t_{\mathrm{A}}(\mathrm{My})$ & $t_{\mathrm{B}}(\mathrm{My})$ & $\Delta t / t_{\mathrm{A}}$ \\
0.014 & 130 & 60 & 0.54 \\
0.021 & 2750 & 5080 & -0.85 \\
0.015 & 480 & 484 & -0.01 \\
\hline
\end{tabular}

reference models for both components in order to determine sensitivity of luminosity of each components to heavy element abundance and find time at which $L(t)=L_{\mathrm{obs}}$. The difference between the times required for the agreement of models with observed luminosities is a function of $Z$. The time differences for some $Z$ values are listed in Table 2 . The time difference between the ages of the component stars is negligibly small when $\mathrm{Z}=0.015$. The age of the system is then $480 \mathrm{My}$. From the calibration of radii around this age, we find that $\alpha_{A}=1.30, \alpha_{A}=1.05$ and $\mathrm{t}=500 \mathrm{My}$. For $\mathrm{X}=0.718$, models with $\mathrm{Z}=0.0127$ give the same and very similar mixing-length parameters: $\alpha_{A}=1.27$, $\alpha_{A}=0.97$. As a result, for different combinations of $\mathrm{X}$ and $\mathrm{Z}$ we find that for also this system mixing-length parameter is an increasing function of stellar mass

In Fig. 1, evolutionary tracks of FL Lyr A and B are plotted in HR diagram. While FL Lyr A is slightly evolved, FL Lyr B is very close to the ZAMS line.

\section{The mixing-length parameter from the eclipsing binary V442 Cyg}

The components of this system are F-type stars. Applying the same method as for FL Lyr, we find that $Z=0.0142$ and age of the system is about $1.73 \mathrm{~Gy}$, for $\mathrm{X}=0.705$. From the calibration of radii, the mixing-length parameters of V442 Cyg A and B are found as $\alpha_{\mathrm{A}}=3.46$ and $\alpha_{\mathrm{A}}=2.29$. Very similar values are found for different combinations of $\mathrm{X}$ and Z. So, for also this system, we obtain that the mixing-length parameter is increasing function of stellar mass. Furthermore, the values of mixing-length parameter for V442 Cyg B is compatible with the value derived from expression for Hyades.

\section{Conclusion}

In order to fit model radii of late type stars to their observed radii, one of possible ways is that the mixing-length parameter is an increasing function of stellar mass around ZAMS. For the later phase of MS evolution, while the same result is found from analysis of the eclipsing binary V442 Cyg, the seismic and and non-seismic constraints of $\alpha$ Cen give contrary results. For the later phases, old eclipsing binaries in old open clusters can be a useful lab (for example, V12 binary system in NGC 188). 


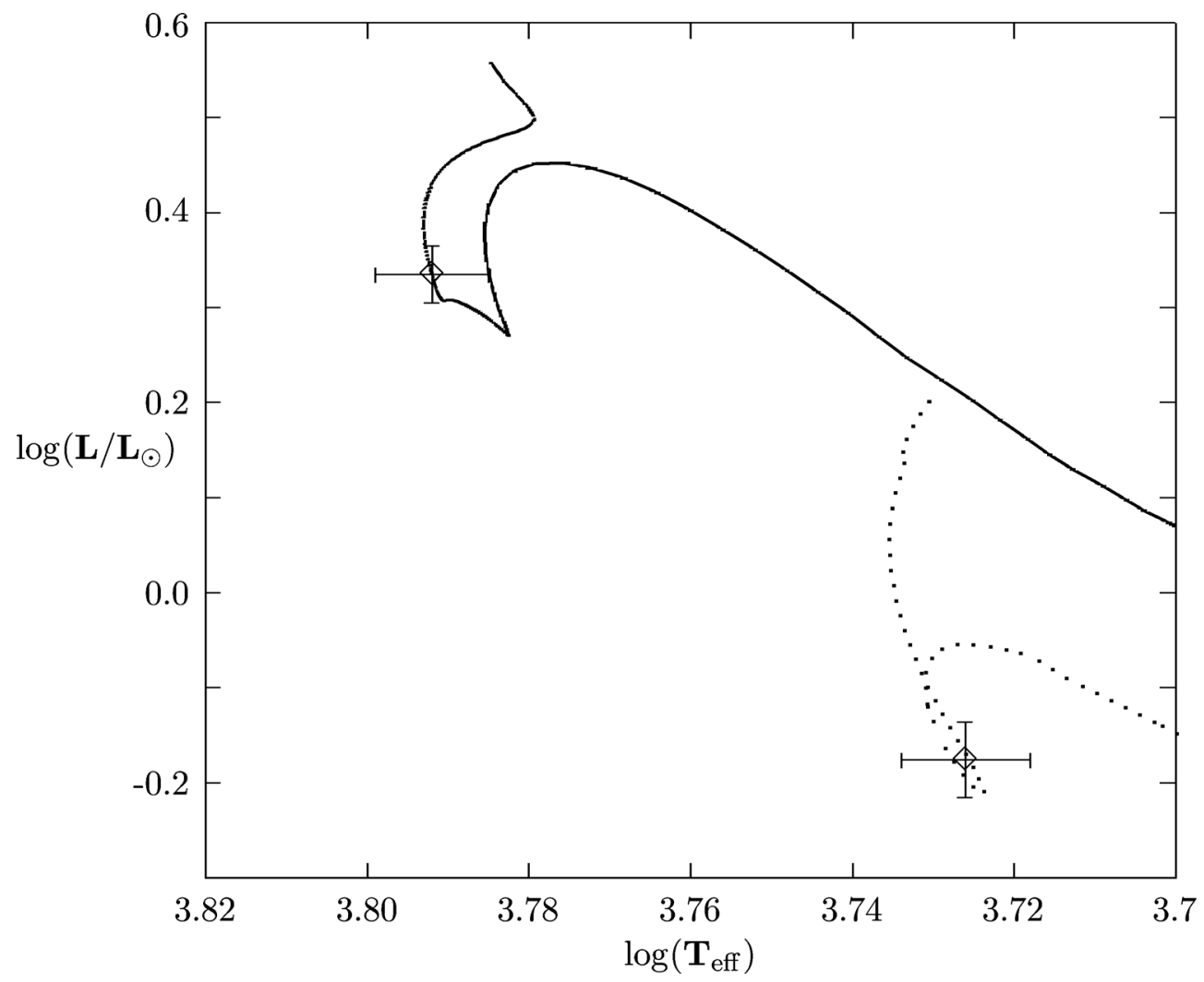

Figure 1. HR diagram for FL Lyr A and B. The models are computed with $\mathrm{Z}=0.015$ and $\mathrm{X}=0.7$. The agreement is obtained at $\mathrm{t}=500 \mathrm{My}$.

\section{References}

Andersen, J. 1991, A $\mathscr{E} A R$ 3,91

Bohm-Vitense, E. 1958, Zs. Ap. 46, 108

de Bruijne, J. H. J., Hoogerwerf, R., \& de Zeeuw, P. T. 2001, A\&A, 367, 111

Lebreton, Y., Fernandes, J., \& Lejeune, T. 2001, A\&A 374, 540

Lejeune, T., Cuisinier, F., \& Buser, R. 1998, A\&AS, 130, 65

Miglio, A., Montalban, J., 2005, A\&A, 441, 615

Pols, O. R., Tout, C. A., Schroder, K-P., Eggleton, P. P., \& Manners, J. 1997, MNRAS 289, 869

Popper, D. 1980, ARAEA 18, 115

Popper, D. 1997, AJ 114, 1195

Yildız, M., 2006, MNRAS, 368, 1941

Yıldız, M., 2007, MNRAS, 374, 1264

\section{Discussion}

ZAHN: When you analyze your binary stars, do you assume the same chemical composition for both components?

YILDIZ: Of course. One of the main assumptions about binary systems is that their component stars have the same initial chemical composition.

LUDWIG: Which presicion do you obtain for the mixing length parameters considering the observational uncertainties (in colors, chemical composition, radii, etc.) 
Remark: Intrinsically, the mixing length parameter is a function of the stellar atmospheric parameters ( $T_{\text {eff }}$, gravity, chemical composition), and perhaps radius if sphericity effects become important. Hence, one should rather use these as independent variables instead of age, mass and luminosity to describe its functional dependence.

YILDIZ: The problem here is that the models of late type stars with standard theory are inadequate to refer observed radii. The considered stars are among the well known stars whose fundamental properties are relatively very accurate. However, for the stars in Hyades, for example, the slopes of main sequence in two intervals used as main constraints on models are very precise. Of course, there are some other possible mechanisms in order to restore the disagreement between the model and observed radii, but mixing-length parameter is perhaps the first among them to test.

Convection is one of the most complicated phenomena in the nature. Regarding this point in mind, the main point here is to show the variability of the mixing-length parameter without any prejudice and use more complicated expression if required. 